\title{
A Cost-Benefit Analysis of Testing for Influenza A in High-Risk Adults
}

\author{
William J. Hueston, MD \\ Joseph J. Benich III
}

Department of Family Medicine, Medical University of South Carolina, Charleston, SC

\begin{abstract}
BACKGROUND Clinical diagnosis and empiric therapy have been strategies for treatment of suspected influenza in high-risk patients, but rapid tests for influenza have been introduced to help confirm cases. The aim of this study was to determine when rapid testing, empiric treatment, or no treatment is most cost-beneficial for high-risk adults with influenzalike respiratory tract illnesses.
\end{abstract}

METHODS We performed a cost-benefit analysis evaluating the comparative advantage of the strategies of empiric therapy, no treatment, or test and treat patients whose tests are positive. The analysis focused on a hypothetical population of patients who are at a high-risk for complications of influenza. Our main outcome was the cost of care for an episode of influenza taken from the human capital perspective.

RESULTS For older anti-influenza drugs (amantadine and rimantadine), rapid testing is not as cost-beneficial as empiric treatment, even when the prevalence of influenza is low. For the neuraminidase inhibitors, there is a narrow window of disease prevalence between $30 \%$ and $40 \%$ where testing is most cost-beneficial. When the disease likelihood is above this window, empiric treatment is preferred. Below this window, no treatment is more cost-beneficial. Even under the most favorable conditions, testing is preferred only for a small range of prevalence rates of influenza.

CONCLUSION When clinicians are planning to use the nonneuraminidase inhibitors to treat influenza, rapid testing is not the most cost-beneficial approach. Even when the more expensive neuraminidase inhibitors will be used, testing has a limited role in managing influenza in high-risk patients.

Ann Fam Med 2004;2:33-40. DOI: 10.1370/afm.34.

\section{INTRODUCTION}

I nfluenza is a common respiratory tract infection that can result in considerable morbidity and mortality for unvaccinated high-risk persons.

Influenza infects approximately 20 to 30 million Americans each year and causes severe suffering and loss of productivity. Overall, influenza causes about 200,000 hospitalizations each year, along with 10,000 to 40,000 deaths. ${ }^{1}$ Although the impact of influenza and influenzalike illness on productivity and health care resource utilization in a working population is great ${ }^{2}$ most morbidity and mortality related to the illness are seen primarily in the elderly and those with underlying respiratory tract conditions. ${ }^{3}$

For patients who have not been vaccinated, antiviral treatments can reduce the risk of complications, ${ }^{4-7}$ but treatment must be started within 48 hours to provide any benefit, and confirming the diagnosis of influenza by viral culture is not practical for clinicians. Consequently, clinicians have had to judge the likelihood of influenza, as opposed to other respiratory tract illnesses, based on clinical probability and initiate empiric therapy.

To address this issue, several new rapid diagnostic tests have been aimed at reducing the time to confirm the diagnosis of influenza. These 
tests identify influenza antigens in nasal and pharyngeal secretions and provide a result in a matter of minutes instead of the days required for culture. The appropriate use of these tests, however, is still undetermined. A previous study looking at healthy adults found that testing for influenza before treatment with a neuraminidase inhibitor was cost-beneficial only when the probability of influenza was between $10 \%$ and $35 \%$, depending on the expected cost savings. ${ }^{8}$ This study did not focus on high-risk persons, for whom the prevention of complications might be beneficial, and did not examine the cost-benefits of testing when using older, less expensive anti-influenza drugs.

The purpose of this study was to evaluate whether testing with treatment of patients with positive tests or an alternative strategy of treating all patients with suspected influenza was more or less costly than symptomatic care for patients at high risk for a complication of influenza. We conducted cost-benefit analyses looking at the incremental cost or savings for each of 4 available anti-influenza drugs based on strategies to treat empirically or to test for influenza and treat patients who have positive results compared with a baseline strategy of no antiviral treatment. In particular, we hoped to define the potential costs (or savings) to patients who elect to take medication for influenza and what effect testing before treatment would have on this cost. Because most of these patients will have to pay out-of-pocket expenses for medication costs, as well as a portion of their testing cost, we believed that these patients should understand the cost and potential benefit of either of these options so that they can decide whether they are obtaining value from treatment or testing.

\section{METHODS}

\section{Population}

We based our analysis on a simulated cohort of 1,000 unvaccinated patients who were at high risk for complications or hospitalization from influenza. This group would include those older than 65 years and those older than 50 years who have chronic obstructive pulmonary disease, asthma, or other chronic respiratory tract conditions; preexisting malignancy; diabetes; or cardiac diseases. The underlying assumption for this analysis was that these patients would complain to their clinicians of respiratory tract symptoms within 48 hours of symptom onset so that they would be candidates for antiviral therapy.

The study focused on those infected during a season in which influenza A strain is predominant. We chose to limit the analysis to influenza A because studies examining the effectiveness at preventing influenza complications with antiviral therapy have been per- formed only with patients who have been infected with influenza A. Given that the ability to avert or reduce the severity of complications and the potential benefit associated with these reductions have not been established for community-acquired influenza B, we could not examine the cost-benefit relationship for patients infected with this strain. Additionally, data from the Centers for Disease Control and Prevention show that between the 1995-1996 and 2001-2002 influenza seasons, influenza $\mathrm{A}$ was the predominant strain in every season. ${ }^{9}$ Even though influenza B did circulate widely late in the 2001-2002 and 1996-1997 seasons, influenza A has carried the largest burden of disease for patients in the United States during the past 7 years.

\section{Perspective}

We performed the analysis from the human capital perspective. This approach considers all incremental costs after the initial visit, including direct costs (such as medications or physician visits) and indirect personal costs (such as lost wages for the patient or the patient's caretaker) and societal costs (such as insurance payments for hospital or outpatient services) associated with the illness regardless of the payer. ${ }^{10}$

Because influenza is a self-limited problem with a relatively brief period of symptoms, we adopted a time horizon of 1 month. During this time, the vast majority of complications should have become evident. With such a short time-horizon, we did not discount future costs.

\section{Variables}

The decision tree used for the study is shown in Figure 1. The following variables were considered in the analysis: the population probability of influenza (ie, the chance that a patient with respiratory tract symptoms will have influenza as opposed to another viral respiratory tract infection), the cost of diagnostic testing, the sensitivity and specificity of diagnostic test, the cost of initial medication, the probability of adverse reaction to medication, the cost of initial clinical visit caused by an adverse drug reaction, the probability of a serious complication, the cost of serious complication, and the cost savings for avoidance of a serious complication. The benefits of drug treatment in patients with influenza were based on additional work productivity associated with an earlier recovery from illness. We did not include the cost of the initial visit to the clinician, because all patients already would have incurred this expense at the time the decision regarding testing or treatment was made. A summary of baseline assumptions for all variables is shown in Table 1.

The cost of the subsequent clinical visits was based on the weighted probability of respiratory tract infections made by a cohort of 22,144 patients in $1996 .{ }^{11}$ 


\section{Figure 1. Decision tree used for the cost-benefit analysis.}

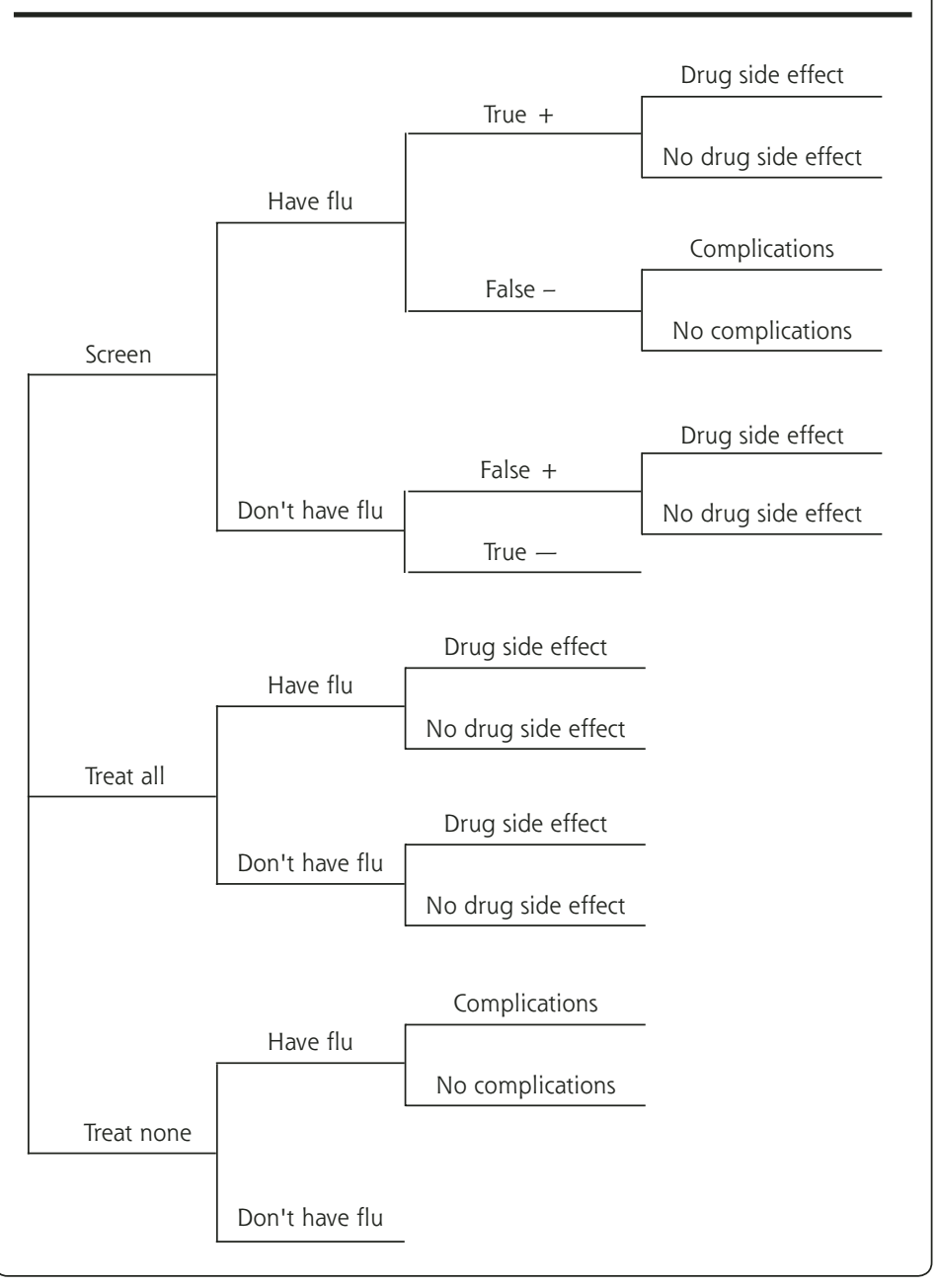

Table 1. Baseline Probability and Cost Assumptions for Influenza: Testing-Treatment Model

\begin{tabular}{lcc}
\hline Variable & $\begin{array}{c}\text { Baseline } \\
\text { Assumption }\end{array}$ & $\begin{array}{c}\text { Sensitivity } \\
\text { Test Range }\end{array}$ \\
\hline Cost assumptions & 20.00 & $5-30$ \\
Cost of diagnostic test, \$ & 177.20 & * \\
Benefits of recovery, \$ & 40.48 & $32.38-48.54$ \\
Additional physician visit for drug reaction, \$ & $8,960.20$ & $7,175-10,763$ \\
Complication with hospitalization, \$ & 10.50 & $8.40-12.60$ \\
Medication costs (full course of therapy) & 24.08 & $19.26-28.90$ \\
Amantadine, \$ & 49.35 & $39.48-59.22$ \\
Rimantadine, \$ & 61.00 & $48.80-73.20$ \\
Zanamivir, \$ & & $50-95$ \\
Oseltamivir, \$ & 72.5 & $80-00$ \\
Probability assumptions & 90.0 & $0-6$ \\
Test sensitivity, \% & 3.0 & $0.3-5$ \\
Test specificity, \% & 0.5 & \\
Probability of drug side effect, \% & & \\
Probability of influenza complication, \% & & \\
\hline * For benefits, a threshold analysis was performed (see Figure 3 and text). & \\
\hline
\end{tabular}

Visit costs for diagnostic codes for influenza (ICD-9 code 487.1), along with streptococcal pharyngitis (ICD-9, 034.0), otitis media (ICD-9, 382.9), acute nasopharyngitis (ICD-9, 466.0), acute sinusitis (ICD-9, 461.9), acute pharyngitis (ICD-9, 462.0), acute tonsillitis (ICD-9, 463.0), acute bronchitis (ICD-9, 466.0), and pneumonia (ICD-9, 486), were combined in a weighted fashion based on their frequency to determine a weighted visit cost for respiratory tract infections as described by Bridges et al. ${ }^{12}$ Costs were then adjusted from 1996 costs to 2002 costs by multiplying the 1996 values times the medical component of the consumer price index to produce a median visit cost per respiratory tract infection visit.

Diagnostic testing costs were based on the average cost of 5 currently commercially available test kits. The average cost data from 2 counsulted sources were used for each test kit. Test kit costs ranged from $\$ 18.00$ to $\$ 24.50$. The average overall cost was $\$ 20.00$. In addition to direct cost, testing requires between 15 and 45 minutes to perform, depending on the test used. ${ }^{12}$ It is unclear, however, how much time is required for direct personnel activity and how much is incubation time. Additionally, batching multiple specimens in a large clinic could reduce the direct test performance time considerably. For those reasons, we did not include any indirect cost to the practice for performing this test beyond the expected reimbursement. We also did not include any indirect cost to the patient, because the brief period of time waiting for the results (15 to $45 \mathrm{~min}$ utes) was unlikely to have any important economic impact.

Sensitivity and specificity data were based on the average findings from studies examining test characteristics. ${ }^{13-21}$ When indicated, test performance characteristics were used from adult patients; when the age of the participants was not specified, summary data were used. The sensitivity of the 5 diagnostic tests ranged from $51.4 \%$ to $92.0 \%$, with an average of $72.5 \%$. Specificities ranged from $82.0 \%$ to $99.0 \%$, with an average of $90.0 \%$.

The cost of medication was based on the cost to a patient for a 5-day course of 
treatment at recommended full doses. The costs of medications were obtained from a community pharmacy and from a national publication. ${ }^{22}$ The 2 sources differed by a range of $\$ 0.45$ to $\$ 6.36$. Rates for adverse drug events were based on the average rates of adverse events from treatment studies in the Pbysician's Desk Reference. ${ }^{23}$ As a baseline, we assumed that each adverse event would prompt an additional physician visit, but we conducted sensitivity analyses described below to examine the effects if fewer patients required additional medical care for their adverse drug reaction.

The probability of serious complications of influenza was based on data reported by the Centers for Disease Control and Prevention. ${ }^{24}$ The cost of serious complications was calculated from the mean cost of a hospitalization in 1996 for influenza. ${ }^{12}$ This cost was adjusted to 2002 dollars by multiplying the values obtained from 1996 times the medical component of the consumer price index.

The benefit of early treatment was based on reports that treatment decreases symptoms 24 hours earlier than no treatment. ${ }^{4,5}$ The economic impact assigned to this benefit was increased productivity based on the patient being able to return to work (or having a caretaker return to work) 1 day earlier. The average cost was based on 8 hours of the 1999 average hourly wage plus benefits for a worker in the United States converted to 2002 dollars. ${ }^{11}$ Admittedly, this assumption could be excessive, because there are no data examining earlier return to work for patients treated with antiviral drugs. Additionally, retired patients might not derive any additional direct economic benefit by recovering 1 day earlier. To address this issue, we conducted sensitivity analyses varying the indirect benefit from $\$ 0$ to a maximum of 1 day of work. In the case where a patient does not have to return to work, this indirect benefit can be construed as the "willingness to pay" to recover 1 day sooner.
Figure 2: Cost-benefit for testing or treatment for different probability of influenza.

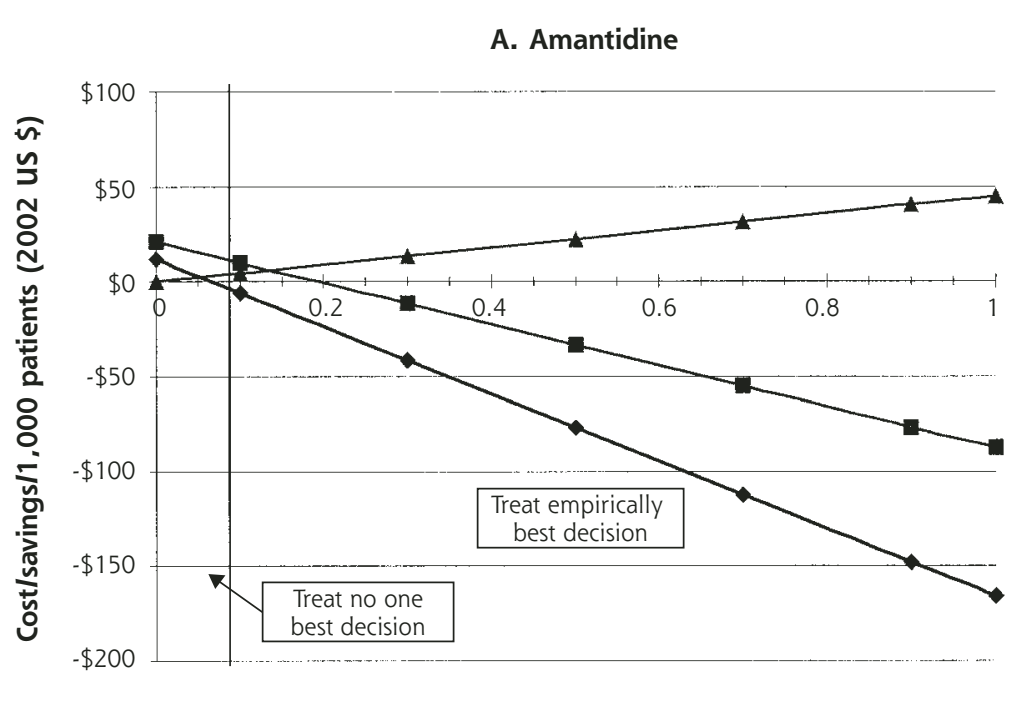

Probability of influenza

B. Rinantidine

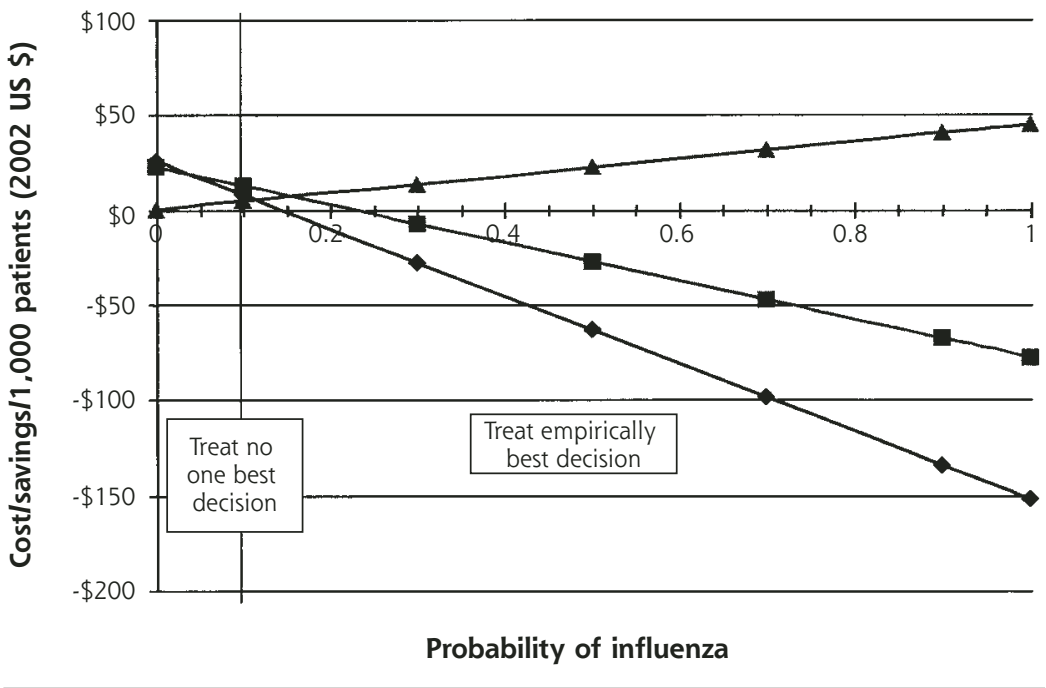

Note: Lines represent cost of illness episode based on the following strategies: Treat all (diamonds), test and treat patient with positive test results (squares), and treat none (triangles).

\section{Analyses}

Analyses were performed using Microsoft Excel spreadsheets One-way sensitivity analyses were performed for all variables over the ranges noted in Table 1 . Two-way sensitivity analyses were performed to examine the impact of changes in the economic benefits over the entire range of pretest probability and test characteristics. These results are presented as threshold analyses representing the point at which the economic value assigned to an earlier recovery alters the decision for the entire range of pretest probabilities. 


\section{Zanamivir}

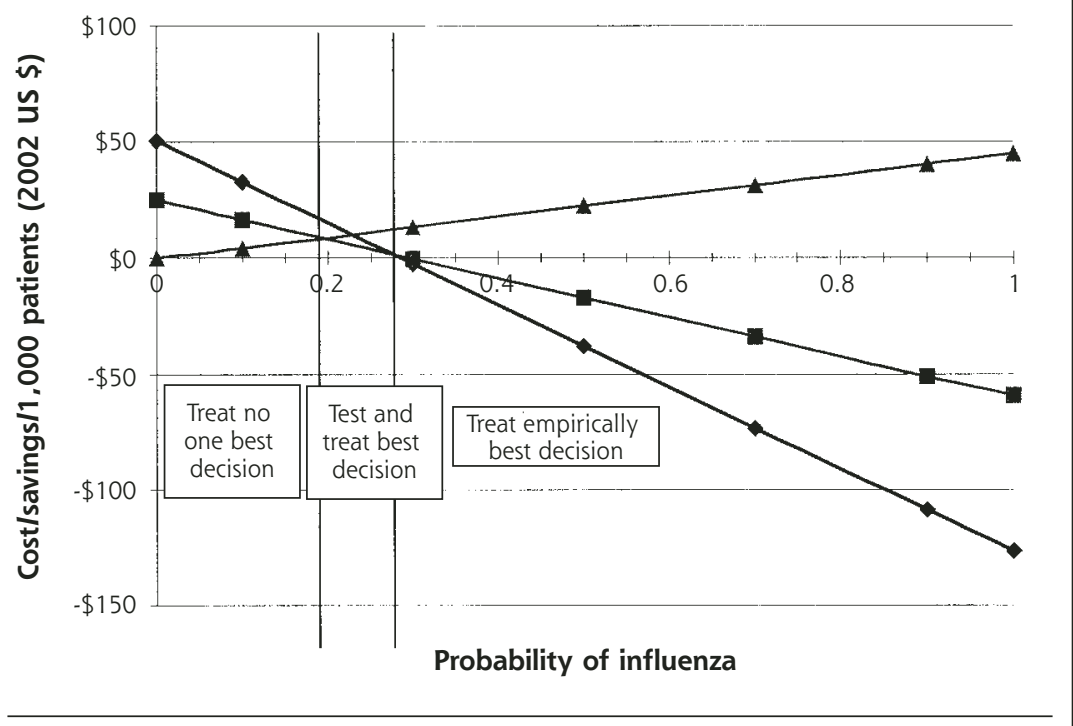

D. Oseltamivir

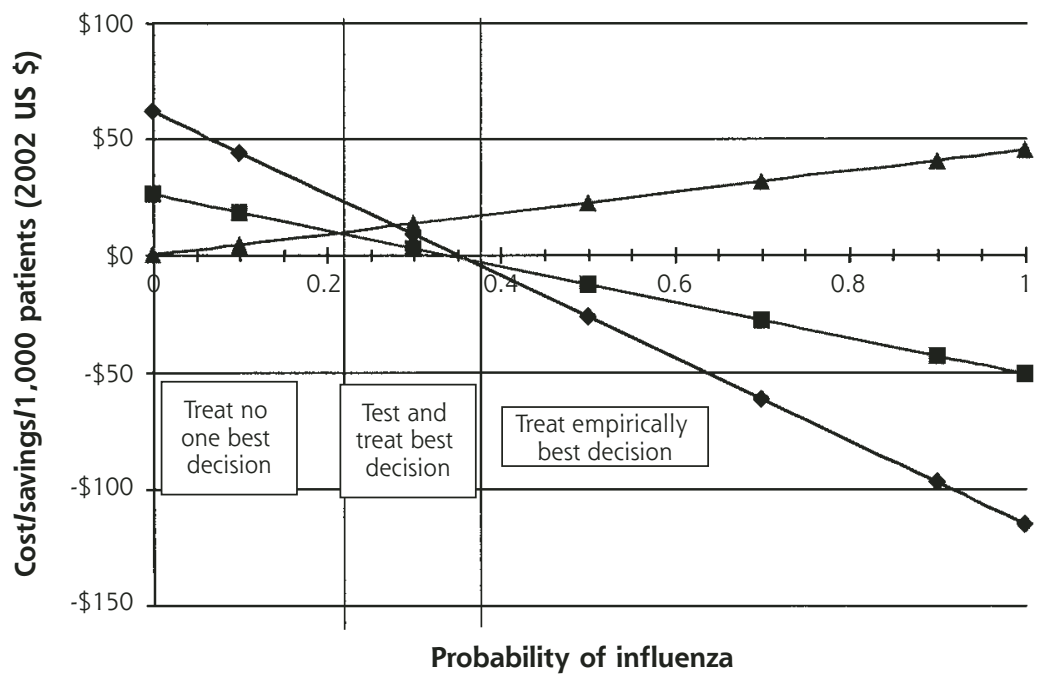

\section{RESULTS}

The costs of each treatment decision for all 4 drugs are shown in Figure 2. Because the selection of the medication influences the cost, individual panels in Figure 2 indicate the costs when amantadine (panel A), rimantadine (panel B), zanamivir (panel C), or oseltamivir (panel D) are used for treatment.

Because the costs involved in the no-treatment strategy are not affected by drug costs, the costs of the no-treatment strategy are the same in all 4 panels. As is noted in Figure 2, when the probability of the patient having influenza is 0 , the no-treatment strategy involves no incremental costs beyond the initial visit. As the probability that the patient has influenza increases, however, the no-treatment strategy becomes more expensive because of the small costs associated with complications and the larger costs caused by lost productivity.

When we focus on the strategy to treat empirically, Figure 2 shows significant cost savings of this strategy compared with no treatment whenever the probability of having influenza is greater than $6 \%$ for amantadine or $34 \%$ for oseltamivir. At $100 \%$ probability of having influenza, compared with no treatment, treat empirically with amantadine produces the highest average saving at $\$ 0.21$ per patient. In the same situation, treatment with ramantadine saves $\$ 0.20$, treatment with zanamivir saves $\$ 0.17$, and treatment with oseltamivir saves $\$ 0.15$ per patient. As the probability of having pneumonia declines, the cost-benefit of treat empirically compared with no treatment decreases.

The test-and-treat strategy also showed cost savings compared with no treatment, which decrease as the probability of influenza falls. As noted in Figure 2 , though, the cost savings associated with test and treat are less than those with the treat empirically strategy in most cases. For amantadine and rimantadine, the test-and-treat strategy never saves more money than the treat empirically strategy for the entire range of population probabilities. For oseltamivir, test and treat appears to be the most cost-beneficial strategy when the probability of influenza is between $22 \%$ and $36 \%$. For zanamivir, test and treat has an even smaller window of cost-benefit, $19 \%$ to $28 \%$. At prevalence rates of less than these cutoffs, it is more cost-beneficial not to treat patients. At rates higher than these, empiric treatment is the most cost-beneficial option. 
Table 2. Most Cost-Beneficial Treatment Strategy for Each Antiviral Drug

\begin{tabular}{lccc}
\hline Drug Prescribed & $\begin{array}{c}\text { No } \\
\text { Treatment } \%\end{array}$ & $\begin{array}{c}\text { Test Before } \\
\text { Treatment } \%\end{array}$ & $\begin{array}{c}\text { Empiric } \\
\text { Treatment \% }\end{array}$ \\
\hline Amantadine & $<5$ & -- & $\geq 5$ \\
Rimantadine & $<11$ & -- & $\geq 11$ \\
Zanamivir & $<19$ & $\geq 19$ but $\leq 28$ & $>28$ \\
Oseltamivir & $<22$ & $\geq 22$ but $\leq 36$ & $>36$ \\
\hline
\end{tabular}

Note: Based on probability that a high-risk patient has influenza: population probability where strategy is most cost-beneficial.

Table 2 summarizes these findings by indicating when each strategy is the most cost-beneficial. The table shows that for amantadine and rimantadine no treatment is best only when the probability of the patient having influenza is fairly low. When the chance that the patient has influenza is higher than this value, empiric treatment is less costly. Testing before treatment was never the least costly strategy when using these drugs. Because of the higher cost for the neuraminidase inhibitors, no treatment was preferable when the probability of having influenza was about $20 \%$. Treat empirically was best when the probability of influenza is better than 1 in 3 . When the probability is between these two values, then testing is the best strategy.

\section{Sensitivity Testing}

We varied a wide number of variables to determine under which circumstances diagnostic testing was optimal. We found that the cost of the test and the test performance characteristics had the largest influence on when testing was cost-beneficial. Yet, even when the cost of the test was decreased to $\$ 1$ and the sensitivity and specificity were set at $100 \%$, the population probability where testing was less expensive than empiric therapy only increased to $57 \%$ for oseltamivir and was even less for zanamivir. Consequently, whenever the probability of the patient having influenza was better than about $50 \%$, empiric treatment remained preferable. For the non-neuraminidase inhibitors, even at these extreme values, empiric treatment always was less expensive than testing.

When the probability of drug side effects and the cost of these side effects were increased, testing became cost-effective at higher disease probabilities. Again, even for the most expensive drug, when drug side effects were set at $10 \%$ and the cost of side effects increased to $\$ 300$ per episode, empiric treatment saved money compared with no treatment when the probability of disease was more than $50 \%$.

Because the risk of complications from influenza are very low, variations in the disease complication rate and complication costs had little effect on the decision to test. As would be expected, increasing the rate of complications made empiric treatment the preferred decision at even lower probabilities of disease. Conversely, as the risk of complications declined or costs decreased, no treatment became more costbeneficial at higher probabilities. The effect of lowering the benefit of reduced symptoms was more pronounced for drugs that cost more and had a lesser effect than it was for less expensive agents.

Finally, we looked carefully at the effects of altering the economic benefits on the decision. Because many patients in the high-risk group might not work, the benefits assigned to increased productivity in the baseline assumptions might be high. For that reason, we determined the threshold value for benefits where treatment would be preferable to no treatment. Figure 3 shows the threshold value for the economic benefit at which either treat empirically (Figure 3A) or test and treat (Figure $3 \mathrm{~B})$ becomes preferred to no treatment. As is evident in Figure 3A, because of the reduction in complications and the low cost of the medications, both amantadine and rimantadine are cost-beneficial compared with no treatment, even when the additional cost is $\$ 0$, providing that the probability of influenza is greater than $50 \%$. Both strategies appear to be favored when compared with no treatment, even when the probability of influenza is greater than $30 \%$ and the patient assigns a benefit of earlier recovery of $\$ 100$ or more.

\section{DISCUSSION}

This analysis shows that the 2 most important factors to consider when deciding whether to treat empirically or test a patient for influenza are the probability that the patient has influenza and the drug that will be used for treatment. When influenza is probable (greater than $50 \%$ chance), empiric treatment is the best strategy regardless of the drug used. When influenza is less likely (20\% to $40 \%$ chance), testing is a cost-beneficial strategy only if the physician plans to use a neuraminidase inhibitor. When the physician plans to use amantadine or rimantadine, testing is more expensive than empiric treatment. In fact, empiric treatment with amantadine or rimantadine even seems to be cost-beneficial when the chance of having influenza is unlikely but still possible ( $5 \%$ to $10 \%$ ). Only when it is fairly certain that the patient does not have influenza is no treatment the most cost-beneficial approach.

An additional finding of this analysis is that unless the probability of having influenza was 0 , neither test- 
ing then treating nor no treatment saved money. Only empiric therapy when the probability of influenza was moderate to high resulted in a decrease in overall costs. The highest cost savings occurred with the use of the least expensive drugs. This finding argues for greater use of these drugs when influenza is likely and the patient is likely to benefit. Our finding that early treatment saves money might encourage patients to seek care early in the course of influenzalike illnesses, when the drugs would still be effective. It should be kept in mind, however, that the absolute cost savings per patient with early treatment are still very small compared with the cost of a physician office visit.

Our results for high-risk patients are similar to those reported by Blitz and coworkers ${ }^{8}$ for healthy adults. In their study looking only at testing when neuraminidase inhibitors were used to treat influenza, testing was cost-beneficial only at lower probability rates for the disease. In an Australian study that focused on high-risk patients, investigators reported that zanamivir treatment resulted in an incremental cost of $\$ 14.20$ (Austra-

\section{Figure 3: Threshold analysis for individual benefit.}

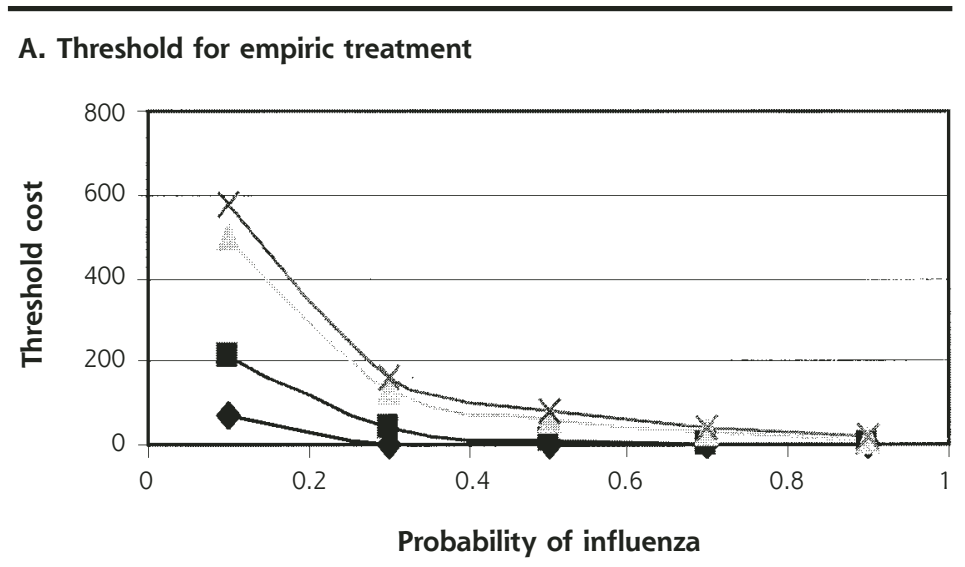

B. Threshold for test and treat

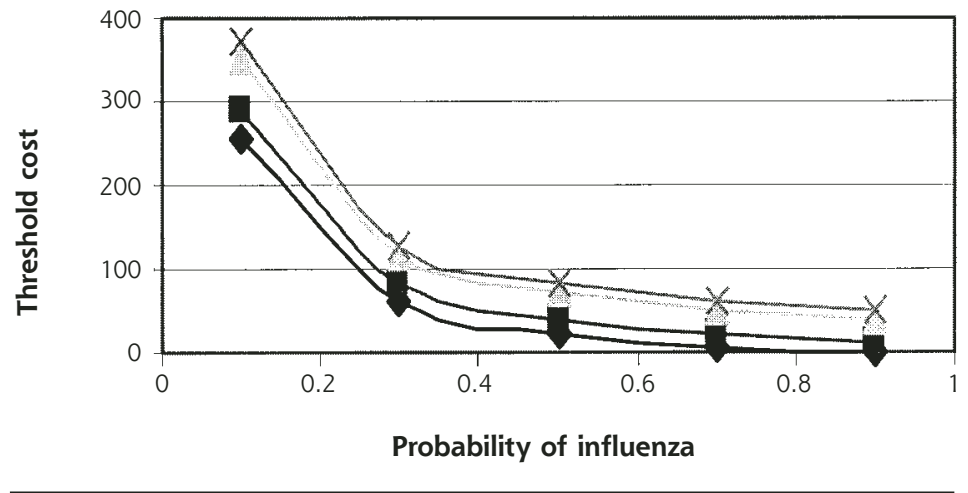

Note: Either empiric treatment (3A) or test before treatment (3B) is preferred to no treatment for amantadine (diamonds), rimantadine (squares), zanamivir (triangle), and oseltamivir $(X)$. lian) for every patient treated. ${ }^{25}$ Treatment with other drugs was not evaluated. While the investigators did not examine the cost-benefit of rapid tests in determining whether the individual has influenza, they did note that treatment without previous testing was likely to be cost-effective when the prevalence of disease was high, as we found in our study.

One limitation of this study is that it is difficult to assign an exact monetary benefit for the accelerated recovery when influenza is treated. We expressed the benefits of early recovery in terms of enhancing worker productivity, but many older patients do not work, so this figure might not be valid. Depending upon the individual, patients might assign a larger or smaller value to feeling better a day sooner. Our threshold analysis showed, however, that when the probability of influenza is greater that $50 \%$ and amantadine or rimantadine is used, empiric treatment is preferable to no treatment even if there is no additional economic benefit beyond the reduction in complications. Only when the chances of influenza become unlikely (less than $30 \%$ ) do the economic benefits needed to make treatment cost-effective become substantial. Consequently, even though we might not be able to assign a firm economic value to early recovery, the threshold for treatment is within a range that patients might be likely to be willing to pay for an earlier recovery.

A second limitation of this analysis is that we assumed that the patient population is homogeneous when, in fact, patients with influenza are not. Those patients at higher risk for complications, such as very old patients with severe chronic obstructive pulmonary disease, are much different from the 50-year-old, healthy, nonsmoking patient. Clinicians still have to gauge the individual risk to a particular patient when making treatment decisions. Even so, the sensitivity analysis suggests that under most circumstances, testing was rarely the most cost-effective strategy for a patient with an influenzalike respiratory tract illness.

Finally, it should be emphasized that this study focused on high-risk patients who had not received influenza vaccination. Even for low-risk patients, influenza immunization is likely to be cost-beneficial. ${ }^{12}$ For individuals who have been vaccinated and still contract influenza, the benefits of pharmacological treatment might differ from our assumptions. 
Also, this analysis should not be construed as suggesting that drug therapy is cost-beneficial compared with routine immunization. Not all persons for whom influenza immunization is indicated are vaccinated, however, and others are unable to receive vaccination because of contraindications. This analysis may help guide testing or treatment decisions for these patients.

\section{To read commentaries or to post a response to this article, see it} online at http://www.annfammed.org/cgi/content/full/2/1/33.

Key words: Influenza; virus diseases; diagnostic techniques and procedures; cost-benefit analysis

Submitted December 3, 2002; submitted, revised, March 25, 2003: accepted April 21, 2003.

Funding support: Partial support for this project was provided by Grant \#5 D16 HP 00008-03 from the Bureau of Health Professions, Health Resources Services Administration.

\section{REFERENCES}

1. Centers for Disease Control and Prevention. Prevention and control of influenza: recommendations of the Advisory Committee on Immunization Practice (ACIP). MMWR Morb Mort Wkly Rep. 2001;50:(RR-41)1-63.

2. Keech M, Scott AJ, Ryan PJ. The impact of influenza and influenzalike illness on productivity and healthcare resource utilization in a working population. Occup Med. 1998;49:85-90.

3. Hak E, Verheij TJ, van Essen GA, Lafeber AB, Grobbee DE, Hoes AW. Prognostic factors for influenza-associated hospitalization and death during an epidemic. Epidemiol Infect. 2001;126:261-268.

4. Treanor JJ, Hayden FH, Vrooman PS, et al. Efficacy and safety of the oral neuraminidase inhibitor oseltamivir in treating acute influenza. JAMA. 2000;283:1016-1024.

5. Jefferson TO, Demicheli V, Deeks JJ, Rivetti D. Amantadine and rimantadine for preventing and treating influenza $A$ in adults. Cochrane Database Syst Rev. 2000;(2):CD001169;10796612.

6. Caserta MT, Hall CB. Antiviral agents for influenza. Pediatr Ann. 2000;29(11):704-711.

7. Monto AS. Moult AB. Sharp SJ. Effect of zanamivir on duration and resolution of influenza symptoms. Clin Ther. 2000;22:1294-1305.

8. Blitz SG, Cram P, Chernew ME, Monto AS, Fendrick AM. Diagnostic testing or empirical neuraminidase inhibitor therapy for patients with influenza-like illness: what a difference a day makes. Am J Manag Care. 2002;8:221-227.

9. Centers for Disease Control and Prevention. Past influenza seasons. Available at: http://www.cdc.gov/nip/flu/flu-seasons.htm. Accessed Feburary 2003.
10. Irwin D, Weatherby LB, Huang WY, Rosenberg DM, Cook SF, Walker AM. Impact of patient characteristics on the risk of influenza/ILIrelated complications. Available at: www.biomedcentral.com/14926963/1/8. Accessed August 2002.

11. Franham PG, Ackerman SP, Haddix AC, Teutsch SM, Shaffer PA, Dunet DO, eds. Prevention Effectiveness: A Guide to Decision Analysis and Economic Evaluation. New York, NY: Oxford University Press; 1996; 15-16.

12. Bridges $C B$, Thompson WW, Meltzer MI, et al. Effectiveness and cost-benefit of influenza vaccination of healthy working adults: a randomized controlled trial. JAMA. 2000;284:1655-1663.

13. Newton DW, Treanor JJ, Menegus MA. Clinical and laboratory diagnosis of influenza virus infections. Am J Man Care. 2000;6:S265-S275.

14. Yamazaki M, Kimura J, Watanabe $S$, et al. Use of rapid detection assay for influenza viral on nasal aspirate specimens. Kansenshogaku Zasshi. 1999;73:1064-1068.

15. Herrmann B, Larsson C, Zweygberg BW. Simultaneous detection and typing of influenza viruses $A$ and $B$ by a nested reverse transcriptionPCR: comparisons to isolation and antigen detection by immunofluorescence and optical immunoassay (FLU OIA). J Clin Microbiol. 2001: 39;134-138.

16. Hulson TD, Mold JW, Scheid D, et al. Diagnosing influenza: The value of clinical clues and laboratory tests. J Fam Pract. 2001:50: 1051-1056.

17. Boivin G, Hardy I, Kress A. Evaluation of a rapid optical immunoassay for influenza viruses (FLU OIA Test) in comparison with cell culture and reverse transcription-PCR. J Clin Microbiol. 2001;39:730-733.

18. Boon AC, French AM, Fleming DM, Zambon MC. Detection of influenza A subtypes in community-based surveillance. J Med Virology. 2001;65:163-170.

19. Yamazaki M, Mitamura K, Kimura K, et al. Clinical evaluation of an immunochromatography test for rapid diagnosis of influenza. Kansenshogaku Zasshi. 2001;75:1047-1053.

20.Schultze D. Thomas Y. Wunderli W. Evaluation of an optical immunoassay for the rapid detection of influenza $A$ and $B$ viral antigens. Eur J Clin Microbiol Infect Dis. 2001;20:280-283.

21. Shimizu $H$. The rapid detection kit based on neuraminidase activity of influenza virus. Nippon Rinsho. 2000;58:2234-2237.

22. Nissen D, ed. Mosby's Drug Consults 2002. St Louis, MO: Elvesier Press, Inc; 2002.

23. Physician's Desk Reference. 55th ed. Montvale, NJ: Medical Economics Company, Inc; 2001.

24. Bridges CB, Fukuda K, Uyeki TM, Cox NJ, Singleton JA. Prevention and control of influenza: recommendations of the advisory committee on immunization practices (ACIP). Morb Mort Week Rep. 2002;51(RR03):1-31.

25. Mauskopf JA, Cates SC, Griffin AD, Neighbors DM, Lamb SC, Rutherford $C$. Cost effectiveness of zanamivir for the treatment of influenza in a high risk population in Australia. Pharmaco Econ. 2000:17:611620. 